\title{
Ein Beitrag zur Kenntnis von Cortis Tätigkeit in Bern
}

\author{
Von Bruno Kisch, New York
}

In seiner Monographie über Alfonso Conti ${ }^{1}$ hat E. Hintzsche versucht, neues Licht auf die wissenschaftliche Tätigkeit Contis zu werfen. Auch er betont jedoch, daß nicht viel Genaues darüber bekannt ist, welcher Art die Untersuchungen waren, die CoRTI unter VALENTINS Leitung in Bern ausgeführt hat. Es mag deshalb eine Notiz von Interesse sein, die mir im Verfolg meines fortgesetzten Studiums über das Leben und die Tätigkeit Valentrss in die Hände fiel ${ }^{2}$, auf die sich vielleicht Contis Bemerkung im Briefe vom 28. Juli 1850 mit bezieht: er schätze sich glücklich, daß er an den physiologischen Experimenten VaLentins teilnehmen durfte.

Unter dem Titel Zur Entwicklungsgeschichte der Fische findet man eine kurze Notiz in Siebold-KölLIKens Zeitschrift für wissenschaftliche Zoologie (Bd. 2, S. 267, 1850) mit dem Untertitel: «Aus einem Schreiben von G. VAlentiN an A. KöLLIKer.» Der genannte Brief ist datiert von Bern, 23. Oktober 1849. Er beginnt mit den Worten: «Was die mit Dr. Corti untersuchten Hechteier, von denen wir mündlich sprachen, betrifft ... .» Eine kurze Beschreibung dieses Teiles der Versuche und Beobachtungen folgt, bei denen es auf die Bewegung des Fischembryonen im Ei ankam, vor allem aber auf die Geschwindigkeit der Dotterdrehung.

VALENTIN war im Verlaufe dieser Untersuchungen die Mißbildung eines Fisches mit Doppelkopf aufgefallen, und er fährt fort: «ConTI und ich fanden später noch mehrere Mißbildungen unter den übrigen ausgeschlüpften Hechtchen.» Es folgt eine kurze Angabe über den Tod dieser durch künstliche Befruchtung erzielten Hechte infolge der Mißbildung des schlauchartig ausgezogenen Herzens, und es werden Angaben über die Pulszahl beider Herzen solcher Mißbildungen und über die Pulszahl der heranwachsenden Embryonen gemacht.

Aus den beiden Stellen, in denen Contr hier erwähnt ist, geht Verschiedenes hervor, das zur Kenntnis seiner Lebensgeschichte beiträgt. Erstens erfahren wir, daß er während der Zeit seines Aufenthaltes in Bern nicht nur, wie schon durch die Veröffentlichung von Hintzsche bekannt ist, über das Flimmerepithel in der Gallenblase von Froschlarven gearbeitet hat, sondern daß er vor Oktober 1849 auch an den Untersuchungen Valentins über Fischembryonen und deren Mißbildungen teilnahm, ferner daß er KöLLIKER schon vor Oktober 1849 (durch VALENTIN) bekannt war.

${ }^{1}$ Berner Beiträge zur Geschichte der Medizin und der Naturwissenschaften, Nr. 3 : Alfonso Corti, 1822-1876. Bern 1944.

2 Bruno Kisch, Gabriel Gustav Valentin. In: Victor Robinson Memorial Volume, S. 193, New York 1948. 
VALENTIN hat ein Jahr später einen ausführlichen Bericht über diese seine Untersuchungen in dem Archiv für physiologische Heilkunde veröffentlicht ${ }^{3}$. In dieser Mitteilung hat er die Mitarbeit CoRTIs an seinen Untersuchungen und Einzelheiten der Zusammenarbeit ausführlich gewürdigt. Er schreibt auf Seite 3 dieser Mitteilungen das folgende, auf CoRTi Bezügliche:

«Eine Reihe von Untersuchungen, welche der durch seine Beobachtungen über die Gefäße der (sic!) Psammosaurus (A. Corti De systemate vasorum Psammosauri grisei, Vindobonae 1847, 4. Auflage) bekannte Dr. Conti über die Entwicklung der Fische auf der hiesigen Anatomie anstellte, gab mir die Gelegenheit, die bald zu beschreibende Doppelmißgeburt des Hechtes eine ziemlich lange Zeit zu verfolgen. Wir bemerkten sie zuerst 102 Stunden nach der künstlichen Befruchtung. Wir konnten sie während der ganzen übrigen Zeit des Eilebens untersuchen.»

Diese Arbeit Valentins ist bemerkenswert, weil er nicht nur die heute anerkannte Möglichkeit betont, die von ihm beobachteten Mißbildungen durch Pincettendruck selbst verursacht zu haben, sondern auch, weil er den Nachweis erbringt, daß derartige Mißbildungen sich aus einer einzelnen befruchteten Keimzelle entwickeln und nicht, wie damals vielfach angenommen wurde, aus dem Zusammenwachsen zweier verschiedener Keimzellen.

Was die Bemerkung Valentins betrifft, daß die Untersuchungen auf der Berner Anatomie angestellt waren, so läßt der Inhalt von VALENTiNs ausführlichem Bericht keine Zweifel darüber, daß sie unter seiner Leitung und unter Mitarbeit von CoRTI durchgeführt wurden. Obwohl VALENTIN erst 1854 nach dem Ausscheiden des Anatomen Theire Direktor der Berner Anatomie wurde, welches Amt er bis 1862 inne hatte ${ }^{4}$, so hatte er doch schon seit der Übernahme der Berner Professur für Physiologie im Jahre 1836 sein Arbeitszimmer im Anatomiegebäude und somit Hausrecht in diesem Institut. Der Anatom Friedrich WILhelm Theile (1801-1879), aus Sachsen-Weimar stammend, ursprünglich Privatdozent und von 1832 an außerordentlicher Professor an der Universität Jena, war bei Gründung der Universität Bern dahin als Anatom berufen worden. Aus der erwähnten Bemerkung geht nicht ohne weiteres hervor, daß CoRTI auch unter THEILE selbst gearbeitet hat, aber es ist nicht ausgeschlossen und jedenfalls auffällig, daß Valentin in seiner Arbeit erwähnt, Contı sei «auf der Anatomie» tätig gewesen. Theile war bis 1853, in welchem Jahre er um seine Entlassung ansuchte ${ }^{4}$, nicht ordentlicher Professor geworden, was Valentin, der gleichzeitig das Fach der pathologischen Anatomie vertrat, seit seiner Berufung nach Bern war. Erst 1854, nachdem der enttäuschte und gekränkte THEILE um seine Entlassung gebeten hatte, wurde er von der Regierung zum ordentlichen Professor befördert und gleichzeitig seinem Entlassungsgesuch stattgegeben ${ }^{4}$.

${ }^{3}$ G. Valentin, Ein Beitrag zur Entwicklungsgeschichte der Doppelmißgeburten. Archiv für physiologische Heilkunde 10, 1-39, 1851.

${ }^{4}$ R. Feller, Die Universität Bern 1834-1934. Bern/Leipzig 1935. 
Daß Corti zu Theile in freundschaftlichen Beziehungen gestanden und wohl auch an den Arbeiten in dessen Institut teilgenommen hat, kann man daraus schließen, daß er in dem ersten der von Hintzsche veröffentlichten Briefe an Valentin in der Nachschrift als ersten unter seinen Berner Bekannten Theile erwähnt, dem er Grüße zu übermitteln bittet.

\section{Goethe und die Urzeugung \\ Von Hubert Erhard, Adelholzen (Bayern)}

Neben Goethes Sterbestuhl liegt heute noch die Bibel und daneben steht ein Teller mit Gartenerde, die er noch an seinem letzten Lebenstag mikroskopisch untersucht hat. Einen weiten Weg müssen wir gehen, um diesen Vorgang zu erklären.

Der älteste griechische Naturphilosoph, Thales, nimmt an, alles Leben entstamme dem Wasser, aus Schlamm. Zu Goethes Zeiten bekämpften sich die Vulkanisten und die Neptunisten; erstere (Cuvier, A.v.HumboLDT) lehrten, die Welt sei durch plötzliche Vulkanausbrüche entstanden, die letzteren, sie sei durch stetige Meeresablagerung gebildet worden (WERner). Goethe war Neptunist. Er ging von dem LeIBNizschen Gesetz der Kontinuität aus, demzufolge es im Reich der Materie wie des Geistes keine plötzlichen Sprünge, sondern nur einen kontinuierlichen Fluß aus Ursache und Wirkung gebe. Dem entsprach die Lehre der Neptunisten.

Nun hatten die griechischen Naturphilosophen vier Elemente angenommen: Wasser, Erde, Feuer, Luft, wobei schon bei ihnen die Vorstellung anzutreffen ist, alles entstünde aus einem polaren Gegensatz; dabei galt ihnen Erde und Wasser als ein solcher Gegensatz.

Als GoEthe 1769/70 in seiner schweren Krankheit die Werke der Alchemisten studierte, fand er bei ihnen die Vorstellung, es gebe «eine hylozoistische, d. h. im Feuchten lebenhaltende Urmaterie, die sogenannte Wassererde» (Spranger). ${ }^{1}$

Im April und Mai 1786 mikroskopiert Goethe eifrig. Der Inhalt eines Heftes, das von Goethes Hand den Titel trägt: Infusionstiere, ist veröffentlicht. ${ }^{2}$ Hier

1 Eduard Spranger, Goethes Weltanschauung, Insel-Verlag, 1946, S. 28. Vgl. auch: Paul WaLden, Zur Problematik der Alchemie und ihrer Ausstrahlungen auf die modernen Naturwissenschaften. In Die Naturwissenschaften, 1948, 35. Jahrgang, Heft 8, S. 228.

${ }^{2}$ Weimarer Sophienausgabe, II. Abt. Band Morphologie, II. Teil, S. 289-304, mit Zeichnungen Goethes S. 305-9. Unter anderem ist GoETHE hier der Entdecker der Hefezellen und der querverlaufenden Zellteilung bei Paramaecium. Aus Infusionen von etwa 20 Objekten (wie getrockneten Steinpilzen, Bier, Roggen, Linsen, Bohnen, Erdäpfeln usw.) erhält er nach mehreren Tagen «Infusionstiere». Paramaecium, Stylonychia, Rotatorien, wie Lepadella, Cyclopiden, Hefezellen, Schimmel, und zeichnet sie erkennbar. Das Folgende in GoEthes Schreibweise. 